\title{
Contrast Information of Digital Imaging in Laser Film Digitizer and Display Monitor
}

\author{
Shih-Chung B. Lo, Jerry W. Gaskill, Seong K. Mun, and Brian H. Krasner
}

\begin{abstract}
A 10-bit or 12-bit gray scale is provided in commercial laser film digitizers. The true contrast resolution on the digitized image within a laser spot area of 200 $\mu \mathrm{m}$ in diameter is limited by both the quantum mottle and instrumentation noise. In this report, we investigated the mean value, standard deviation, and adjecent pixel correlation coefficient on a calibrated step wedge film with two laser digitizers. The results were disappointing, because we found that the evaluated contrast information is inferior to the manufacturers' specifications. On the output side, the brightnesses of different gray levels from a clinical monitor were measured with a narrow angle luminance probe and evaluated by a brief human perception study. In addition, the implications for teleradiology applications are discussed. (C) 1990 by W.B. Saunders Company.
\end{abstract}

KEY WORDS: contrast resolution, correlation coefficient, luminance, teleradiology.

$\mathbf{I}^{\mathrm{N}}$ MPLEMENTATION of a complete digital radiology department entails the conversion and storage of film images in a picture archiving and communication system (PACS). In addition, the development of teleradiology requires highspeed communication channels and a highquality front-end system for image acquisition and image display. It is important to ensure that the film image conversion process does not lose clinically useful information. Four major types of digitizer are currently available: (1) video cameras; (2) charge coupled device (CCD) cameras; (3) drum scanners, and (4) laser scanners. The overall performance of the laser scanner has been reported in the literature. ${ }^{1-3}$ In this report, we present the results of an investigation of some advanced physical performance parameters associated with the gray value information in two commercially available laser film digitizers. On the display side, we assumed that a monitor is a proper clinical viewing screen. The quality of display monitors varies. The value of luminance versus gray value was measured and is reported in the second part of this paper.

\section{EVALUATION OF CONTRAST INFORMATION}

Film and Laser Film Digitizers

A du Pont calibrated film with step wedge was digitized on the two commercial laser film digitiz- ers (which will be referred to as LFD1 and LFD2). Each step wedge on the calibrated film was labeled with the optical density (OD) provided by manufacturer and was confirmed by the densitometer (X-RITE model 301) in this laboratory. The laser digitization spots of the LFD1 and the LFD2 are $200 \mu \mathrm{m}$ and $175 \mu \mathrm{m}$ in diameter. LFD1 generated 12-bit gray values and LFD2 generated 10-bit gray values. Both laser digitizers are designed as high-speed, fineresolution densitometers for a two-dimensional flat object (specifically for a film). However, the laser digitizer measures each transmission rate of the light in the spot in term of an OD that is stretched to a higher scale for integer output called gray value or gray level. Theoretically, the stretched optical densities (gray values) should be proportional to those ODs obtained by a calibrated densitometer. Twelve-bit and 10-bit gray values stand for measured stretched OD of a film digitizer ranging from 0 to 4,095 and from 0 to 1,023 , respectively. In this study, we further scaled all original 10-bit gray values of LFD2 by a factor of 4 in order to compare the 12-bit gray values received by LFD1. The mean value and standard deviation were obtained from the center part $(10 \times 10$ pixels area $)$ of each step region, which covers the OD ranging from 0.20 to 3.41 . The deviation of the digitization process includes both quantum noise (inherent in the calibrated film) and instrumentation noise. The standard

From the Department of Radiology, Georgetown University, Washington, DC; the Department of Radiology, George Washington University, Washington, DC; and AT \& $T$ Bell Laboratories, West Long Branch, $N J$.

Supported in part by the US Army Medical Research Acquisition Activity, Contract No. DAMD 17-86-C-6145.

The views, opinions, and/or findings contained in this document are those of the authors and should not be construed as an official Department of Army position, policy, or decision unless so designated by other documentation.

Address reprint requests to Shih-Chung B. Lo, PhD, Department of Radiology, Georgetown University Medical Center, 3800 Reservoir Rd NW, Washington, DC 20007.

(c) 1990 by W.B. Saunders Company.

0897-1889/90/0302-0006\$03.00/0 
deviation is calculated using the formula:

standard deviation

$$
=\left[\left[\Sigma_{\mathrm{x}} \Sigma_{\mathrm{y}}(\mathrm{f}(\mathrm{x}, \mathrm{y})-\text { mean })^{2}\right] /(\mathrm{N}-1)\right]^{1 / 2}
$$

where $\mathrm{N}$ is the number of pixels in the area.

In addition, the within-a-step correlation coefficient of the residual image, which is defined below, was calculated. This is a measure of the suspected noise in the original digitized image. The adjacent pixel correlation coefficient is given by

correlation coefficient $=$ covariance $/$ variance

where

covariance (for row correlation)

$$
\begin{aligned}
= & {\left[\Sigma_{\mathrm{x}} \Sigma_{\mathrm{y}}(\mathrm{f}(\mathrm{x}, \mathrm{y})-\text { mean })\right.} \\
& \cdot(\mathrm{f}(\mathrm{x}-1, \mathrm{y})-\text { mean })] /(\mathrm{N}-1)
\end{aligned}
$$

covariance (for column correlation)

$$
\begin{aligned}
= & {\left[\Sigma_{\mathrm{x}} \Sigma_{\mathrm{y}}(\mathrm{f}(\mathrm{x}, \mathrm{y})-\text { mean })\right.} \\
& \cdot(\mathrm{f}(\mathrm{x}, \mathrm{y}-1)-\text { mean })] /(\mathrm{N}-1)
\end{aligned}
$$

and

$$
\text { Variance }=\left[\Sigma_{\mathrm{x}} \Sigma_{\mathrm{y}}(\mathrm{f}(\mathrm{x}, \mathrm{y})-\text { mean })^{2}\right] /(\mathrm{N}-1)
$$

Images were first scaled down by a factor of 2 (number of low-bits trimmed). The resultant values were then rounded to the nearest integer, multiplied by the scaling factor, and subtracted from the original image to get a residual image.

$$
r(x, y)=f(x, y)-[\{N\}(\{F\} f(x, y) / S F)] \cdot S F
$$

where SF equals the scaling factor, \{ \}represent an operator, $\{F\}$ indicates a floating point operation, and $\{\mathbf{N}\}$ represents the nearest integer operation.

The standard deviation measurement would provide a parameter associated with a signal-tonoise ratio produced by the digitizer for each step wedge area. The correlation coefficient of the residual image would determine how many most significant bits of the pixel values are correlated. Throughout these studies, as a quality assurance procedure, the user will be able to assess how much the contrast information is obtained by a digital device.

\section{Display Monitors}

The output information of the gray level was the primary subject in the display monitor study. Many of the monitors, the majority of them Display Tek monitors, are currently used in the clinical environment within the Radiology Department of Georgetown University Hospital. We have investigated the luminance response from each increment of gray level (0 to 255). A Display Tek Lambda II Monochrome Display monitor was used in the luminance study. The experiment was conducted by (1) software generated uniform gray value areas $\left(\approx 1\right.$ inch $\left.^{2}\right)$ displayed on the monitor, and (2) luminance measurements obtained from the Tektronix J16 Digital Photometer with a J6523 $1^{\circ}$ Narrow Angle Luminance Probe. The luminance is a measurement of the number of photons flux per unit area. Foot-Lambert (fL) is a luminance unit referring to the number of photons flux measured $1 \mathrm{ft}$ away from the light source of a Lambert. Although the monitor can only handle an 8-bit value ( 0 to 255 ) per pixel for display, the frame buffer is capable of holding up to a 12-bit value per pixel in this particular display system. Hence, the image with a pixel value higher than 8-bit is remapped to 8-bit for display.

\section{RESULTS}

\section{Digitized Gray Values and Their Deviations}

The calibrated OD and the corresponding mean gray value are shown on Fig 1 for LFD1, LFD1 with the diffuser, and LFD2. It was noted that the regular LFD1 and LFD2 gray scale

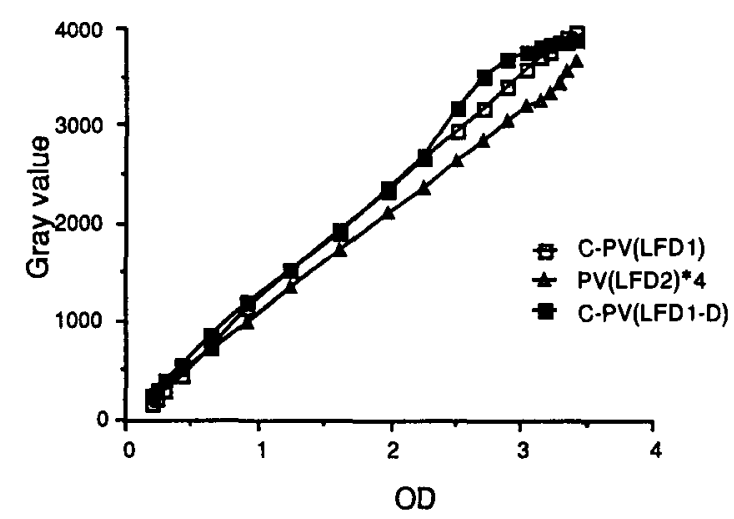

Fig 1. Mean gray value versus calibrated $O D$ for two laser scanners, LFD1 and LFD2. LFD1-D (LFD1 with diffuser). 


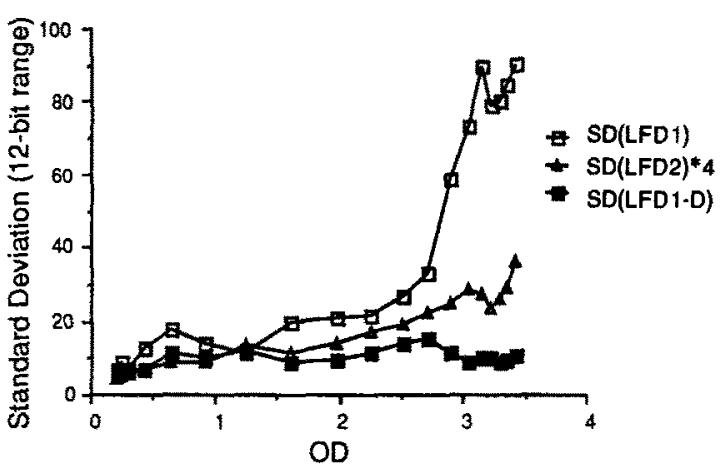

Fig 2. Standard deviation versus calibrated OD for two laser scanners, LFD1 and LFD2. LFD1-D (LFD1 with diffuser).

demonstrated good linearity response versus OD. The LFDI with the plastic diffuser shows nonlinearity in the OD region from 2.48 to 3.41 . It was found that the regular LFD1 produced larger standard deviations in $\mathrm{OD}$ values higher than 2.48. In the worst case, there was a standard deviation of 91 at a mean gray level of 3,968.

The purpose of the plastic diffuser is to suppress the noise level in the digitization process. With the plastic diffuser, the standard deviation was limited in value to the range of 6 to 16 across the entire step wedge OD range (0.20 to 3.41$)$, the standard deviation obtained from LFD2. Figure 2 illustrates these results. Theoretically, the mean to standard deviation ratio on the high OD step should be higher than on the low OD step. This is due to the combination effect of the photon Poisson distribution and the Hurter and Driffield (H\&D) curve response of a film/screen system. ${ }^{4,5}$ The explanation for the excessive deviation is the instrumentation noise, which, in fact, is the major noise source for the high OD range.

The results of the correlation coefficient study are shown on Table 1 and Table 2 for the regular
LFD1 and LFD2, respectively. In this report, a correlation coefficient of less than 0.1 was considered an insignificant correlation. It was noted that for LFD1 the significant correlation coefficients were obtained only after the five least significant bits were trimmed for LFD1. No significant correlation was found for LFD1 with $O D$ equal to 3.41 through the entire residual value correlation study. Some correlation was found for LFD2 after trimming only the three to four least significant bits. These results indicate that only the eight most-significant-bit (MSB) contains contrast information based on the calibrated film for both laser digitizers. Similar results were obtained with a larger area $(40 \times 100$ pixels) and in two chest radiographs. ${ }^{6}$ These results are consistent with the result obtained from the standard deviation study described previously.

\section{Luminance Response Versus Gray Value on a Display Monitor}

The research on the display monitor was focused on the luminance emitted from a display monitor that was expected to deliver accurately distinguishable gray values in the image viewed by the radiologist. The results are shown in Fig 3. A corresponding luminance versus low gray value and the summary of luminance increments are shown in Table 3. It was noted that for gray values less than 20 , luminance increments were $0.01 \mathrm{fL}$ for each gray value increment ( 1 of 256$)$. This indicates that the monitor emitted different luminance values for each gray value change as measured by the luminance probe. However, this does not imply that the human eye is capable of perceiving a different brightness at each gray value change.

Table 1. Residual Value Correlation Coefficient of the Step Wedge (LFD1, 12-Bit Output)

\begin{tabular}{|c|c|c|c|c|c|c|}
\hline \multirow{3}{*}{$\begin{array}{l}\text { No. of Bits } \\
\text { Trimmed }\end{array}$} & \multicolumn{6}{|c|}{ Optical Density } \\
\hline & \multicolumn{2}{|c|}{0.25} & \multicolumn{2}{|c|}{1.61} & \multicolumn{2}{|c|}{3.41} \\
\hline & Row & Column & Row & Column & Row & Column \\
\hline 1 & -0.173 & -0.107 & -0.077 & 0.027 & -0.129 & 0.088 \\
\hline 2 & 0.041 & -0.100 & -0.059 & -0.112 & 0.092 & 0.055 \\
\hline 3 & 0.003 & -0.001 & -0.017 & -0.078 & 0.009 & 0.008 \\
\hline 4 & -0.084 & -0.001 & 0.054 & -0.150 & 0.028 & -0.042 \\
\hline 5 & 0.361 & -0.071 & 0.056 & -0.044 & -0.109 & 0.072 \\
\hline 6 & 0.379 & -0.063 & 0.011 & 0.005 & 0.047 & -0.002 \\
\hline 7 & 0.692 & -0.143 & 0.209 & 0.043 & -0.102 & -0.008 \\
\hline 12 & & & 0.175 & 0.149 & -0.441 & -0.081 \\
\hline
\end{tabular}


Table 2. Residual Value Correlation Coefficient of the Step Wedge (LFD2, 10-Bit Output)

\begin{tabular}{|c|c|c|c|c|c|c|}
\hline \multirow{3}{*}{$\begin{array}{l}\text { No. of Bits } \\
\text { Trimmed }\end{array}$} & \multicolumn{6}{|c|}{ Optical Density } \\
\hline & \multicolumn{2}{|c|}{0.25} & \multicolumn{2}{|c|}{1.61} & \multicolumn{2}{|c|}{3.41} \\
\hline & Row & Column & Row & Column & Row & Column \\
\hline 1 & 0.015 & -0.020 & -0.110 & -0.114 & -0.109 & 0.000 \\
\hline 2 & -0.002 & 0.034 & 0.075 & 0.047 & -0.087 & 0.096 \\
\hline 3 & 0.145 & -0.056 & -0.096 & 0.027 & 0.088 & -0.133 \\
\hline 4 & 0.295 & 0.117 & 0.200 & 0.182 & 0.055 & 0.101 \\
\hline 5 & & & 0.171 & 0.313 & 0.221 & -0.100 \\
\hline 6 & & & 0.443 & 0.234 & 0.431 & -0.101 \\
\hline 7 & & & & & 0.848 & 0.133 \\
\hline
\end{tabular}

In the preliminary visual perception study, three nonradiologists and one radiologist could not perceive the difference between luminances of $0.11 \mathrm{fL}$ and $0.12 \mathrm{fL}$ or between $0.12 \mathrm{fL}$ and $0.13 \mathrm{fL}$. The small square of $0.20 \mathrm{fL}$ surrounded by a $0.11 \mathrm{fL}$ background on the Society of Motion Picture and Television Engineering (SMPTE) pattern were barely resolvable. The window-level program, which is available in most image processing systems and which was proposed by Pizer for viewer-controller mapping, ${ }^{7}$ was used to remap the luminance response curve. The two regions were then much more resolvable (with $\mathrm{W} / \mathrm{L}=280 / 115$ ). The original and remapped luminance curve for the low gray level value is shown in Fig 4. This method could be a simple solution for viewing subtle low gray value change. A more sophisticated approach using automatically determinable mapping was also proposed by Pizer et al. ${ }^{8}$

\section{DISCUSSION}

We conducted a series of studies on the contrast information in a laser digitized image. Our measurements have been made as a quality

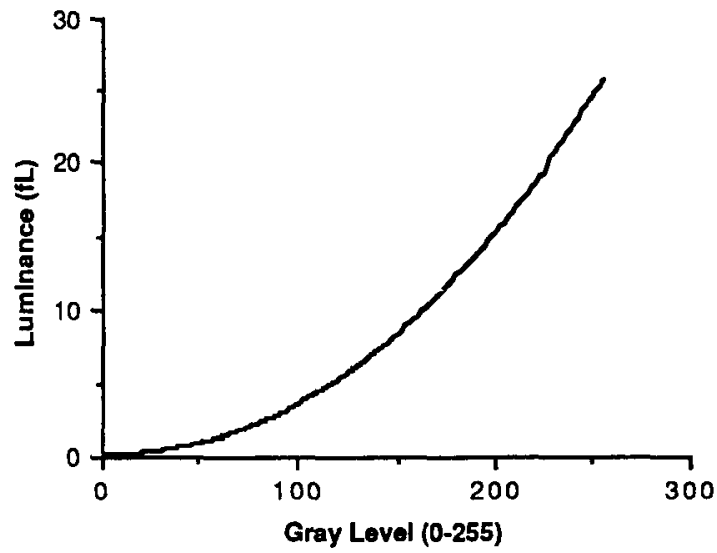

Fig 3. Luminance versus gray value from the monitor. assurance procedure to evaluate the effective contrast information using a digital device. The results of the standard deviation and correlation coefficient studies indicated that the image information exists in the 8-bit range for a $200-\mu \mathrm{m}$ spot in both local and global areas. Ten-bit and 12-bit gray level information were not found through digitization of a calibrated film. The excessive deviation results in part from the low transmission of light in the high OD region received in the detecting device of the laser digitizer. This is an inherent physical problem in the film digitization process. A slight increase of the deviation would be understood by and acceptable to the user. A high gray level output value accompanied by a large standard deviation and low correlation measurement in the flat area is not acceptable. The high gray value data would increase the required storage and would slow down the image communication speed in either a PACS or a teleradiology system. In fact, exaggerated gray value output was found in magnetic resonance imaging (MRI) applications. ${ }^{9}$ Because the film digitizer produces much more data per image than a MR system, its impact on a PACS is more pronounced.

The laser scanner has been the most costeffective of these four types of digitizer for the following reasons ${ }^{1-3}:$ (1) its high resolution,

Table 3. Luminance Values in the Low-Gray-Level Range

\begin{tabular}{ccc}
\hline Gray Value & Luminance (fL) & $\Delta \mathrm{L} / \Delta \mathrm{G}$ (fL) \\
\hline $0-21$ & $0.11-0.30$ & 0.01 \\
$22-41$ & $0.32-0.72$ & 0.02 \\
$42-53$ & $0.75-1.11$ & 0.03 \\
$54-69$ & $1.15-1.80$ & 0.04 \\
$70-82$ & $1.85-2.47$ & 0.05 \\
$83-93$ & $2.53-3.15$ & 0.06 \\
$94+$ & $3.2+$ & \\
\hline
\end{tabular}

NOTES. Original gray level range, 0 to $255 . \Delta \mathrm{L} / \Delta \mathrm{G}$ denotes luminace change per gray value increment. 


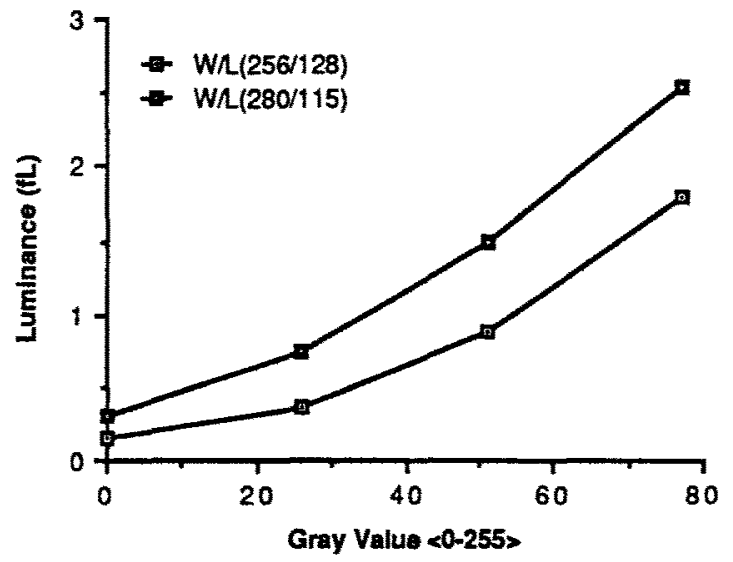

Fig 4. Luminance versus gray value for two different window lovel settings.

$2,000 \times 2,500 \times 10$ bits or $2,000 \times 2,500 \times 12$ bits for $14 \times 17$ inch film; (2) its accurate spatial linearity; (3) its reasonable modulation transfer function (MTF) and contrast frequency response; and (4) its good gray scale linearity in the 8-bit range. In addition to the quality assurance of the physical performance of the digitizer, several factors related to the clinical operation need to be addressed: (1) automation of film feeding; (2) adjustable OD range; (3) demography interface or built-in text recognition intelligence; (4) digitization speed (currently 6 to 40 seconds/film is reported); and (5) interface to a PACS. In the data management and communication system, the display and communication strategy will affect the system performance, and thus, clinical utility. Two types of image transmission features are proposed: hierarchical image data base with a specific compression/decompression datamanipulation design, ${ }^{10}$ and priority of transmission based on area of interest. The problem with the second type of strategy is the difficulty in obtaining prior knowledge of the area of interest.

The display monitor is inferior to the conventional film/alternator setup in a variety of areas: (1) low spatial resolution; (2) low gray value dynamic range; and (3) slow image-display speed. However, if the monitor is incorporated with an electronic image processing device, the potential image enhancement could be useful in the clinical environment. In this paper, we have demonstrated that the clinical monitor does emit small increments of luminance $(0.01 \mathrm{fL} / \mathrm{gray}$ level $)$ in the low gray value region ( 0 to 21$)$. Larger luminance gradient increments were found beyond gray value of 21 and were shown both in Fig 3 and Table 3 . From this preliminary visual study, we found that $.01 \mathrm{fL}$ increment per gray level is not perceived by the human eye. However, $0.02 \mathrm{fL} /$ gray level is a barely resolvable increment. Luminance changes with higher than $0.02 \mathrm{fL} /$ gray level are resolvable. A possible solution to this type of luminance response is the use of window and level mapping techniques to move the entire gray scale to a region of higher incremental luminance. The drawback in this method is that the dark area luminance must shift from a low luminance to a slightly higher luminance (background luminance was changed from $0.11 \mathrm{fL}$ to $0.20 \mathrm{fL}$ in this experiment), which is not perceived as black. Pizer and his colleagues have proposed using a look-up table for each monitor in order to convert the luminance response curve to an ideal response curve. The problem of this technique is that the conversion has to be performed either by the manufacturer or in the low-level software display routine. Neither is easily controlled by the user.

\section{REFERENCES}

1. Lo SC, Taira RK, Mankovich NJ, et al: Performance characteristics of a laser scanner and laser printer system for radiological imaging. Comput Radiol 10:227-237, 1986

2. Takeuchi H, Taira RK, Huang HK, et al: Preliminary experience with a laser scanner and printer system for radiological image. SPIE Proc, PACS III 536:65-71, 1985

3. Vranck J, Strul B: Performance evaluation of a laser digitizer. SPIE Proc Med Imaging 767:524-528, 1987

4. Macovski A: Medical Imaging Systems. Englewood Cliffs, NJ, Prentice-Hall, 1983

5. Fundamentals of Radiography. Rochester, NY, Eastman Kodak Company, 1980

6. Lo SC, Krasner BH, Mun SK: Noise impact on error-free image compression. IEEE Trans Med Imaging 1990 (in press)

7. Pizer SM: Intensity mappings: Linearization, imagebased, user-controlled. SPIE Proc Display Technol II 271:2227,1981

8. Pizer SM, Johnston RE, Zimmerman JB, et al: Contrast perception with video displays. SPIE Proc PACS for Medical Applications 318:223-230, 1982

9. Stewart BK, Lo SC, Huang HK: Gray level dynamic range in magnetic resonance imaging. SPIE Proc Med XIV/PACS IV 626:189-194, 1986

10. Lo SC, Mun SK: Data compression for radiology image display with visual directory. SPIE Proc Med Imaging II 914:1203-1208, 1988 